\title{
Keeping at-risk youth at the center: lessons learned from a community-based participatory research Photovoice project in Taiwan
}

\author{
https://doi.org/10.37719/jhcs.2020.v2i2.rna003
}

\author{
VICTOR HSIAO, BA 1 \\ https://orcid.org/0000-0002-2409-6436
}

\author{
SUNYA CHEN, BA² \\ https://orcid.org/0000-0002-3811-2038
}

MELLISSA WITHERS, Ph.D., MHS 1

https://orcid.org/0000-0001-7754-0206

${ }^{1}$ Keck School of Medicine, University of Southern California, Los Angeles, California, USA

${ }^{2}$ Taiwan group home (unnamed for confidentiality)

\section{Corresponding author's email: victor.y.hsiao@gmail.com}

\section{Abstract}

Youth in the foster care and juvenile justice systems have numerous unmet health needs and long-term negative health outcomes. Photovoice is a qualitative research method in which participants produce photographs and narratives to communicate their perspectives. While Photovoice has been used in various contexts relating to at-risk youth, no known study has been conducted among youth in the foster care or juvenile justice systems. However, numerous challenges exist for the inclusion of at-risk youth in research. Thirteen youth from a group home in Taiwan for teenage boys in the foster care and juvenile justice systems participated in this yearlong study which utilized a strengths-based approach to examine resiliency, their needs, and sources of support. After receiving in-depth training, participants spent three months taking photos and writing accompanying narratives relating to the research questions. Then, via facilitated individual and group discussions, participants selected photo-narrative pairings relating to five key themes, which were then used in exhibitions for local stakeholders. This article describes nine key lessons learned to keep at-risk youth at the center of future similar research studies through protecting, representing, and empowering them: 1) consider ethical challenges, 2) identify community partners, 3) develop mutual trust with participants, 4) use symbolism, 5) have a strengths-based approach, 6) allow participants to direct the process, 7) maximize time to develop participants' introspective skills, 8) disseminate study results widely, and 9) include participant empowerment as a key objective. Conducting research with at-risk youth is challenging but vital to identifying ways society can best support them. Photovoice 
remains a meaningful way for marginalized communities to articulate their needs and share their experiences and perspectives. Recognizing and addressing logistical and ethical challenges early can ultimately lead to more impactful studies for at-risk youth both individually and systemically.

Keywords: Adolescent Health, Foster Youth, Vulnerable Populations, Community-Based Participatory Research

\section{Introduction}

The term "at-risk youth" is defined as youth with an increased likelihood of unsuccessfully transitioning to adulthood (Koball et al., 2011). Youth in the foster care and juvenile justice systems have numerous unmet health needs and long-term negative health outcomes (Ahrens et al., 2014; Coffey et al., 2003). More research is needed to inform policies meant to address these youth's unique needs.

Top-down research is often employed when studying vulnerable youth. Although this approach can increase awareness of their challenges and needs, it is often much less illuminating than research in which youth participate and describe their own lives and priorities (Barman-Adhikari et al., 2019). Community-based participatory research (CBPR) is a framework that includes community members in the research process and has been widely used to examine health disparities (Wallerstein \& Duran, 2006). CBPR among at-risk youth has been associated with positive outcomes, including increased self-efficacy and confidence, a desire to engage in service work, and decreased risky behavior (Garcia et al., 2014). Photovoice, a specific form of CBPR in which participants use photography and narratives to communicate their unique perspectives, has been used in various contexts with at-risk youth, including adolescents who are migrant workers, substance users, homeless, or from low-income and minority backgrounds (Barman-Adhikari et al., 2019; Bashore et al., 2017; Brazg et al., 2011; Kovacic et al., 2014; Yu et al., 2019).

However, numerous challenges, both logistical and ethical, must be considered when conducting qualitative research among marginalized populations, especially with CBPR. Research that discloses incriminating behavior and experiences of illness can be problematic, especially within small communities because of how disclosure may impact participants' relationships with community members (Teti et al., 2012). Research targeting disadvantaged groups can also lead to increased labeling, discrimination, and stigmatization (Walsh et al., 2008). With youth in the foster care and juvenile justice systems, their age and institutionalized status make it extremely difficult to have opportunities to work with them directly (Jeffords, 2007). It is also hard to longitudinally engage or study youth from these backgrounds because of home environment instability, limited engagement with educational settings, and numerous transitions (between family members, foster homes, and schools) (Barnert et al., 2014; Heath \& Priest, 2016). 
Due to these challenges, youth are often not included in the research meant to benefit them.

Even when researchers seek to connect with these youth, trust may be more difficult to establish given these youth's difficult backgrounds. In qualitative studies, especially participatory research, developing trust before starting a study is critical to the study's success but is also a time-consuming process (Sprague et al., 2019). Teens may avoid conflict or differences in opinions to allow for decisions to be made more quickly, so researchers must be prepared to have a "critically curious standpoint" to avoid results becoming overly simplistic or merely reaffirming assumptions and generalizations (Dovey-Pearce et al., 2019). Participatory research also requires researchers to be open to the idea of multiple truths and leave behind a position of superiority, consciously seeking to minimize power differentials (Sprague et al., 2019). Furthermore, these youth often have decreased communication and literacy levels, due to factors such as schooling disturbances, and have low selfconfidence regarding their educational and communication levels (Bryan et al., 2007; Hopkins et al., 2016). These youth, however, best understand their lived realities and thus it is imperative to include them in the research process.

Unique challenges further arise when studies are conducted internationally and crossculturally. Research is often limited by available resources, especially time and money (Morse et al., 2007; Pischke et al., 2017). Gaining approval from ethics review committees may be a lengthy process, especially if approval from multiple countries is required and if committees have different ethical standards and protocols. International research teams may also encounter misunderstandings, scheduling delays, and poor coordination (Goddard et al., 2006; Pischke et al., 2017). If researchers, especially foreigners, do not share the same culture and are seen as outsiders by local participants, mistrust and misunderstandings may arise (Pischke et al., 2017). These issues may jeopardize the validity and trustworthiness of the data.

Research on at-risk youth is uncommon due to these reasons and international qualitative studies with this population are even more scarce. Currently, in Taiwan, the only known qualitative study that explores the perspectives and views of at-risk youth is the study that is the basis for the lessons learned in this paper (Hsiao et al., 2019). The objectives of this article are to describe the lessons learned from conducting a one-year community-based participatory research study among at-risk teenage boys.

\section{Methods}

\section{Setting}

This Photovoice study was conducted in Taiwan at a group home for teenage boys in the foster care and juvenile justice systems. All 26 boys at the group home during the study period were verbally invited to participate by the co-investigator and group home staff. Participant responsibilities and ethical concerns were communicated to participants and legal guardians before signing the 
consent forms. Monthly suicidal ideation screenings were implemented to ensure participant safety. Ethics approval was granted by National Taiwan University and the University of Southern California.

The on-site co-investigator was an ethnically Taiwan-Cantonese-American, Mandarinspeaking, US medical student who had previously volunteered at the group home for several weeks. The two study facilitators were a guidance counselor and social worker who had worked at the group home for more than ten years. Facilitator and participant training was adapted from one of the coinvestigators' previous work and existing Photovoice manuals (Blackman \& Fairey, 2007; Palibroda et al., 2009).

\section{Training and Data Collection}

The project's two main research questions were "how do at-risk youth view their lived experiences" and "how do these experiences relate to health and wellness?" To begin, weekly group trainings were held for two months which emphasized photography techniques, the use of symbolism, and ethical conduct. Participants then spent three months in data collection taking photos in response to the research questions and prompts, including topics such as sources of support, ways society can better support youth with similar backgrounds, and effective ways to relieve stress. After this data collection phase, each participant selected his top ten photos and wrote accompanying narratives. All participants had regular individual meetings with the research facilitators to discuss their work and address any questions or concerns.

\section{Data Analysis and Dissemination}

Through facilitated discussions, the participants sorted the top photos and narratives into key themes that they felt represented their individual and collective experiences and views. This work was then shared with local stakeholders through a total of 16 exhibitions in Taiwan. Additional presentations were given at various international conferences. Resource guides were also disseminated at exhibitions with tips for volunteering, effective ways to work with teenagers, and a list of local volunteer opportunities.

\section{Findings}

The results of this study are published elsewhere (Hsiao et al., 2019). Below are nine key lessons learned and recommendations to keep future similar CBPR studies focused on at-risk youth through protecting, representing, and empowering them.

1. Consider ethical challenges.

2. Identify community partners.

3. Develop mutual trust with participants. 
4. Use symbolism.

5. Have a strengths-based approach.

6. Allow participants to direct the process.

7. Maximize time to develop participants' introspective skills.

8. Disseminate study results widely.

9. Include participant empowerment as a key objective.

\section{Discussion}

\section{Consider ethical challenges}

The ethics approval process is critical to protecting participants but can be very lengthy due to the age and vulnerable status of youth. Starting early can prevent study delays. Gaining approval from two separate ethics review committees for this study required more than six months. Because neither committee was familiar with Photovoice, numerous in-person and email discussions helped the committee members to better understand how the study protocol ensured participant safety and dignity. For example, photos and narratives were blurred or edited to prevent identification of participants or described subjects and were individually reviewed with participants before inclusion in the exhibits to ensure that the work still matched and conveyed the participants' original intentions.

Identifying appropriate legal guardians for consent forms was also challenging because guardians either didn't understand the consent forms or were unreachable (due to incarceration or recent death) and/or because social workers were uncertain who to contact as the guardian. The difficulties with the consent process highlighted deeper systemic issues relating to families and the child welfare system. The ethical challenges faced when working with this population must be considered to ensure they are protected in the research process but should be discussed and addressed early to minimize study delays.

\section{Identify community partners}

For any community-based study, especially if working internationally, having invested local facilitators and community members involved from the beginning is vital; they will have a deeper understanding of the local population, norms, cultures, and administration. Having local facilitators familiar with the youth allowed us to elicit genuine perspectives without causing re-traumatization and disclosure of information they might later regret sharing.

Many youths had limited experiences with success, delayed gratification, and/or long-term planning. As such, having facilitators familiar with the participants was critical to sustaining participants' motivation, including identifying photography locations participants would enjoy and keeping friend groups together. Nonetheless, as expected, attrition occurred so it was helpful to have 
facilitators present the option of withdrawing but encourage them to complete the study.

The inclusion of local facilitators also led to greater opportunities to share the participants' work and research findings by establishing connections with other interested community stakeholders and identifying presentation opportunities.

\section{Develop mutual trust with participants}

In qualitative research, establishing a relationship of trust, and building rapport between the researchers and participants is critical, but is even more important with this population as many atrisk youth have insecurities, decreased trust, and/or past traumatic experiences. The study setting was selected because the onsite co-investigator had previously spent numerous weeks at the group home. The established relationship and regular interactions with participants allowed us to better ask questions, encourage them when they became frustrated, and give prompts when participants were unsure about what to photograph or how to express themselves.

Creating a safe space for a discussion led to more meaningful and open dialogue. Encouraging participants to discuss ideas or thoughts that they felt might be unfeasible or extreme also led to fruitful discussions about ways to support these youth. Establishing mutual trust was critical to ensuring that shared views and ideas were the youth's genuine perspectives.

\section{Use symbolism}

Participants' described topics were emotionally, and sometimes legally, sensitive. As such, symbolism was central to how topics and subjects were depicted in the participants' photographs. Thinking abstractly and utilizing symbolism were less familiar to participants, due to a combination of disturbances in the participants' schooling and decreased emphasis on these areas in the local education system. As such, many examples and practice exercises were included during the study's training and data collection phases.

Using symbolism allowed for the circumvention of the logistical challenge of photographing certain subjects, such as those that were legally sensitive or related to the past. Symbolism also helped to protect the participants' identities, thereby ensuring their safety and dignity. Symbolism in the photographs further elevated participants' voices as photographs did not directly depict the described subjects but rather represented the ideas and emotions being expressed by the participants (Creighton et al., 2018). The creativity and abstract depiction of certain themes and experiences also provided distance from painful experiences while making the work more compelling than if words were used alone (Kantrowitz-Gordon \& Vandermause, 2016). 


\section{Have a strengths-based approach}

Much of the research on the lived experiences of at-risk youth focus on past trauma, including family dysfunction, substance use, and histories of abuse or neglect. Inevitably, during discussions, participants shared negative past experiences, which were included in the study results. However, for both the purpose of empowering the study participants as well as seeking to identify possible solutions to the issues that are well-established in the literature, a strengths-based approach was used. We aimed to identify the participants' perceived sources of support, the aspects of their lives that had led to the development of resiliency, and their hopes and dreams for the future. In this process, we found that participants not only identified positive areas of their lives that they had previously not reflected on but also grew as they discovered new areas that they could continue to develop. Participants also learned from each other's insights during group discussions, which helped them realize that although they had had challenging pasts, they were not alone.

\section{Allow participants to direct the process}

Although at times we wanted to step in so that results better aligned with our views, giving participants autonomy throughout the study resulted in findings that more closely reflected participants' perspectives and increased participants' empowerment and self-esteem. During differences in opinions, we asked questions to stimulate discussion and avoided altering participants' decisions. We also used both individual and group discussions to promote diversity of ideas.

The photo sorting process required several different approaches. Most participants readily answered questions about their own lives but had more difficulty imagining other participants' perspectives and identifying common themes between photos and their shared histories and experiences. These challenges may be the result of participants' lower reading comprehension levels and the local education system's lesser emphasis on creative and critical thinking. Furthermore, numerous insightful comments came from quieter, but very reflective, individuals but sometimes only if they were given the space or asked to speak. Participants enjoyed seeing each other's photos but thought the sorting process was tedious and mentally tiring. We needed to prompt participants to explore and articulate the meaning of photos, beyond what was simply depicted. All photos and narratives were reviewed and approved by participants before the exhibitions.

\section{Maximize time to develop participants' introspective skills}

Introspection is learned and typically difficult among teenagers but even more so among atrisk youth. Many participants had difficulty writing their narratives due to factors including rarely being asked to express their opinions, lower communication levels resulting from constant disturbances in their schooling, and searching for "the right answer" since the local education system emphasizes memorization and less creativity and critical thinking. Understanding the participants' subculture but not belonging to it allowed us to approach the study with unique perspectives. During the introspective 
process, it was clear that the study provided an opportunity for the participants to see the value of and articulate previously unshared perspectives and experiences. Some participants preferred written (versus verbal) communication because of the distance and space it provided, as participants could work at their own pace to craft their narratives without facing our immediate reactions.

Knowing the challenges of introspection, we maximized the time for training, data collection, and data analysis, allowing us to get to know participants better and allowing them to explore the research questions in detail. This helped to ensure that they produced their best work without time constraints. In the three months of data collection, some participants felt like they did their best work at the beginning when they had the most momentum. But many others' best work was near the end as they grew more familiar with the process. One-on-one meetings with facilitators were frequent and kept short to account for participants' shorter attention spans.

\section{Disseminate study results widely}

We spent much time discussing systemic issues affecting at-risk teenage boys in Taiwan and what dissemination methods would most greatly impact community perspectives on these youth and potentially lead to policy change. Highlighting Photovoice's visual component and identifying invested local stakeholders increased our study's reach.

Working closely with community partners interested in the target population and who have the power to make the change is critical. We were fortunate to meet community partners who connected us to various organizations and institutions that were interested in the research findings and actively exploring ways to better serve this population. In the end, findings were presented at various international conferences and 16 exhibitions around Taiwan, and the work was featured in the newspaper and on television. Presentation sites included local schools, universities, hospitals, government offices, and group homes. Making presentations in non-academic settings accessible to the general public is key to promoting change within the community. Attendee feedback included developing greater empathy and understanding of at-risk youth, affirming the need for but also the rarity of hearing youth perspectives, seeing that fully supporting at-risk youth requires a multidisciplinary approach, and being impressed that the youth produced all the photos and narratives themselves. Providing resource guides (lessons learned and ways to get involved) can encourage meaningful change and attendees to get involved.

\section{Include participant empowerment as a key objective}

Empowerment is central to Photovoice but, like social or policy change, is a long-term process. Providing participants with autonomy throughout the research process allowed them to have control over the direction and results of the study. Individual meetings allowed for personalized feedback and safe spaces for voicing questions and concerns, while group discussions fostered increased conflict resolution skills. We also ensured that all participants had work in the exhibits, even 
though some participants were more skilled photographers than others. We communicated feedback from presentations to participants to make them feel engaged in the process even though ethical restrictions prevented their attendance.

Empowerment can be measured through individual surveys that collect quantitative and qualitative data about each step of the study, including assessment of what participants learned, behavior and attitudinal changes of participants, causes for these changes, and how the study compared to pre-study expectations. Our survey was based on a framework of degrees of empowerment: 1) does the choice exist? 2) does a person make that choice? and 3) will that choice result in the desired result? (Alsop \& Heinsohn, 2005).

Participants' post-study surveys were overwhelmingly positive. They asked for a longer study period with more training and photo outings. Participants felt they developed new insights and perceived changes in themselves after reflecting on and sharing previously unvoiced experiences and feelings. These insights and changes included slowing down to think before acting, increased understanding of the relationship between behavioral choices and health, new ways to express themselves and more courage to do so, motivation to try harder in school, increased emotional stability and knowledge of stress relief techniques, greater acceptance of the past, increased perceived ability to overcome obstacles, feeling valued and having increased self-esteem, and more hope for the future. Some found this yearlong study to be particularly challenging, especially with introspection and abstract thinking. As a result, the exhibits were especially meaningful and gratifying to them as they saw the results of their yearlong commitment and perseverance.

Some participants felt that they did not gain better connections with other participants. The absence of increased connection might relate to these youth's high barrier to trust but could alternatively reflect that participants already had a high level of connectedness with each other since they live together. Some participants also indicated they did not feel like they developed advocacy skills, citing their inability to attend the exhibitions. But they did feel that the co-investigator was acting as their advocate. Empowerment is a long-term process and thus this study is meant to only play a part in promoting empowerment among the participants. Examples of participant feedback are included in Table 1.

\section{Table 1. Examples of Post-Study Survey Feedback from Participants}

"At first it was very boring. But afterward, I found that each photo was an opportunity to express myself...My biggest takeaway was better understanding my inner thoughts and how to express myself. I gained this through the teachers' guidance and taking time to reflect." -Kenny* (14 years old, grew up with an abusive father and came to the group home after running away from home)

"I have decided I don't need to be so shy and can courageously express my thoughts... The study was too short...At first, it was difficult to select [my top] photos but then I found it simple and interesting because I saw that different things have their masterpieces...I feel that the exhibit can allow more people to hear 
and see our real thoughts."

-Joseph (17 years old, entered the juvenile justice system due to the theft)

"There was a big contrast between my pre-and post-study expectations. I never thought so many people would like my work... [Seeing the finished work] gives me a real sense of accomplishment...This activity can be run again to allow the younger boys to express their thoughts. If there are similar activities in the future, everyone can take the opportunity to participate."

-Will (15 years old, came to the group home due to domestic abuse at home)

*All names are pseudonyms

\section{Limitations}

Photovoice has been criticized for often simply creating awareness at the individual level and not leading to social justice amelioration or transformation (Sanon et al., 2014). Through the various exhibitions and news coverage, increased awareness and attitudinal changes occurred at both the individual and community levels. Many issues identified by the study were family-level or even greater systemic ones which will require much greater investment outside the scope of this study.

\section{Implications}

Conducting international CBPR with at-risk youth is not without its challenges. However, data generated directly by participants can provide unique insights that could be overlooked in traditional research methods that do not include them as an integral part of the research process. The Photovoice methodology proved to be a powerful and meaningful way to engage youth to tell their own stories and identify sources of strength and support, all of which was shared with local stakeholders to promote more awareness and community support.

\section{Conflict of Interest}

The authors have no conflict of interest to disclose.

\section{Acknowledgment}

We thank our community partners (especially Dr. Chang-Chuan Chan and Dr. Shu-Sen Chang from National Taiwan University, Dr. Chaucer C.H. Lin and Shu Mei Chuang from Hualien Tzu Chi Hospital, and Hsiao-Chu Tseng from Taipei Veterans General Hospital Yuli Branch) for helping 
to organize the original study's numerous presentations and exhibitions. Furthermore, we would like to thank the following conferences for allowing us to share our work through various presentations: International Human Trafficking and Social Justice Conference, American Academy of Pediatrics National Conference, and American Public Health Association Annual Meeting. Lastly, thank you to the staff and boys at the group home for your participation and support throughout the study.

\section{Funding}

The study was funded by the Keck School of Medicine Dean's Research Scholarship.

\section{Author Contribution}

Victor Hsiao: conceptualization of the study, data curation, formal analysis and investigation, funding acquisition, project administration, and writing of the manuscript. Sunya Chen: data curation, investigation, and project administration. Mellissa Withers: conceptualization of the study, formal analysis, and writing of the manuscript.

\section{References}

Ahrens, K. R., Garrison, M. M., \& Courtney, M. E. (2014). Health Outcomes in Young Adults From Foster Care and Economically Diverse Backgrounds. Pediatrics, 134(6). https://doi.org/10.1542/peds.2014-1150

Alsop, R., \& Heinsohn, N. (2005). Measuring Empowerment in Practice: Structuring Analysis and Framing Indicators. World Bank, Poverty Reduction and Economic Management Network, Poverty Reduction Group.

Barman-Adhikari, A., DeChants, J. P., M. Brydon, D., Portillo, A., \& Bender, K. (2019). On the fringes: How youth experiencing homelessness conceptualize social and economic inequality-A Photovoice study. Journal of Community Psychology, 47(4), 924-942. https://doi.org/10.1002/jcop.22164

Barnert, E. S., Perry, R., Azzi, V. F., Shetgiri, R., Ryan, G., Dudovitz, R., Zima, B., \& Chung, P. J. (2014). Incarcerated Youths' Perspectives on Protective Factors and Risk Factors for Juvenile Offending: A Qualitative Analysis. American Journal of Public Health, 105(7), 1-7. https://doi.org/10.2105/AJPH.2014.302228

Bashore, L., Alexander, G. K., Jackson, D. L., \& Mauch, P. (2017). Improving health in at-risk youth through Photovoice. Journal of Child Health Care, 21(4), 463-475. https://doi.org/10.1177/1367493517734391

Blackman, A., \& Fairey, T. (2007). The Photovoice Manual: A guide to designing and running participatory photography projects.

Brazg, T., Bekemeier, B., Spigner, C., \& Huebner, C. E. (2011). Our Community in Focus: The Use of Photovoice for Youth-Driven Substance Abuse Assessment and Health Promotion. Health Promotion Practice, 12(4), 502-511. https://doi.org/10.1177/1524839909358659 
Bryan, K., Freer, J., \& Furlong, C. (2007). Language and communication difficulties in juvenile offenders. International Journal of Language \& Communication Disorders, 42(5), 505-520. https://doi.org/10.1080/13682820601053977

Coffey, C., Veit, F., Wolfe, R., Cini, E., \& Patton, G. C. (2003). Mortality in young offenders: a retrospective cohort study. BMJ (Clinical Research Ed.), 326(7398), 1064. https://doi.org/10.1136/bmj.326.7398.1064

Creighton, G., Oliffe, J. L., Ferlatte, O., Bottorff, J., Broom, A., \& Jenkins, E. K. (2018). Photovoice Ethics: Critical Reflections From Men's Mental Health Research. Qualitative Health Research, 28(3), 446455. https://doi.org/10.1177/1049732317729137

Dovey-Pearce, G., Walker, S., Fairgrieve, S., Parker, M., \& Rapley, T. (2019). The burden of proof: The process of involving young people in research. Health Expectations. https://doi.org/10.1111/hex.12870

Garcia, A. P., Minkler, M., Cardenas, Z., Grills, C., \& Porter, C. (2014). Engaging Homeless Youth in Community-Based Participatory Research. Health Promotion Practice, 15(1), $18-27$. https://doi.org/10.1177/1524839912472904

Goddard, T., Cranston, N., \& Billot, J. (2006). Making It Work: Identifying the Challenges of Collaborative International Research. International Electronic Journal for Leadership in Learning, 10(1), 1-5. http://ecite.utas.edu.au/62354

Heath, R. A., \& Priest, H. M. (2016). Examining experiences of transition, instability, and coping for young offenders in the community: A qualitative analysis. Clinical Child Psychology and Psychiatry, 21(2), 224-239. https://doi.org/10.1177/1359104515581715

Hopkins, T., Clegg, J., \& Stackhouse, J. (2016). Young offenders' perspectives on their literacy and communication skills. International Journal of Language \& Communication Disorders, 51(1), 95-109. https://doi.org/10.1111/1460-6984.12188

Hsiao, V., Chen, S., \& Withers, M. (2019). Understanding perspectives and lived experiences of at-risk Taiwanese teenage boys through photovoice. Journal of Global Health Science, 1(2). https://doi.org/10.35500/jghs.2019.1.e40

Jeffords, C. R. (2007). Gaining Approval From a Juvenile Correctional Agency to Conduct External Research. Youth Violence and Juvenile Justice, 5(1), 88-99. https://doi.org/10.1177/1541204006295163

Kantrowitz-Gordon, I., \& Vandermause, R. (2016). Metaphors of Distress. Qualitative Health Research, 26(8), 1031-1043. https://doi.org/10.1177/1049732315575729

Koball, H., Dion, R., Gothro, A., Bardos, M., Dworsky, A., Lansing, J., Stagner, M., Korom-Djakovic, D., Herrera, C., \& Manning, A. E. (2011). Synthesis of Research and Resources to Support at-Risk Youth. https://www.acf.hhs.gov/opre/report/synthesis-research-and-resources-support-risk-youth

Kovacic, M., Stigler, S., Smith, A., Kidd, A., Vaughn, L., Kovacic, M. B., Stigler, S., Smith, A., Kidd, A., \& Vaughn, L. M. (2014). Beginning a Partnership with PhotoVoice to Explore Environmental Health and Health Inequities in Minority Communities. International Journal of Environmental Research and Public Health, 11(11), 11132-11151. https://doi.org/10.3390/ijerph111111132

Morse, W. C., Nielsen-Pincus, M., Force, J. E., \& Wulfhorst, J. D. (2007). Bridges and barriers to developing and conducting interdisciplinary graduate-student team research. Ecology and Society, 12(2).

Palibroda, B., Krieg, B., Murdock, L., \& Havelock, J. (2009). A Practical Guide to Photovoice: Sharing Pictures, Telling Stories, and Changing Communities. In Prairie Women's Health Centre of Excellence. http://www.pwhce.ca/photovoice/pdf/Photovoice_Manual.pdf

Pischke, E. C., Knowlton, J. L., Phifer, C. C., Gutierrez Lopez, J., Propato, T. S., Eastmond, A., de Souza, T. M., Kuhlberg, M., Picasso Risso, V., Veron, S. R., Garcia, C., Chiappe, M., \& Halvorsen, K. E. (2017). Barriers and Solutions to Conducting Large International, Interdisciplinary Research Projects. 
Environmental Management, 60(6), 1011-1021. https://doi.org/10.1007/s00267-017-0939-8

Sanon, M.-A., Evans-Agnew, R. A., \& Boutain, D. M. (2014). An exploration of social justice intent in photovoice research studies from 2008 to 2013. Nursing Inquiry, 21(3), 212-226. https://doi.org/10.1111/nin.12064

Sprague, L., Afifi, R., Ayala, G., \& El-Nasoor, M. L. (2019). Participatory praxis as an imperative for healthrelated stigma research. BMC Medicine, 17(1), 32. https://doi.org/10.1186/s12916-019-1263-3

Teti, M., Murray, C., Johnson, L., \& Binson, D. (2012). Photovoice as a community-based participatory research method among women living with HIVIAIDS: ethical opportunities and challenges. Journal of Empirical Research on Human Research Ethics 7(4), 34-43. https://doi.org/10.1525/jer.2012.7.4.34

Wallerstein, N. B., \& Duran, B. (2006). Using Community-Based Participatory Research to Address Health Disparities. Health Promotion Practice, 7(3), 312-323. https://doi.org/10.1177/1524839906289376

Walsh, C. A., Hewson, J., \& Shier, M. (2008). Unraveling Ethics: Reflections from a Community-based Participatory Research Project with Youth. The Qualitative Report, 13(3), 379-393. https://nsuworks.nova.edu/tqr/vol13/iss3/4

Yu, C., Lou, C., Cheng, Y., Cui, Y., Lian, Q., Wang, Z., Gao, E., \& Wang, L. (2019). Young internal migrants' major health issues and health-seeking barriers in Shanghai, China: a qualitative study. BMC Public Health, 19(1), 336. https://doi.org/10.1186/s12889-019-6661-0

\section{About the Authors}

Victor Hsiao, BA, is a medical student at the Keck School of Medicine of USC. He has a passion for global health and working with medically underserved communities. Victor's dedication to supporting and further understanding at-risk youth led to his selection as a Keck School of Medicine Dean's Research Scholar and him conducting a yearlong Photovoice study examining the social determinants of health among Taiwanese teenage boys in the foster care and juvenile justice systems.

Sunya Chen, BA, has been a guidance counselor for over 14 years at a Taiwan group home for teenage boys in the foster care and juvenile justice systems. She was originally a special education elementary school teacher and came to the group home to be a volunteer tutor. Seeing the students' need for a loving family environment, she decided to stay at the group home as a full-time guidance counselor, taking on the responsibility of overseeing the students' academics and care.

Mellissa Withers, Ph.D., MHS, is an associate professor of clinical preventive medicine at the Keck School of Medicine of USC. She also directs the Global Health Program of the Association of Pacific Rim Universities, a non-profit network of 55 leading research universities in the region. Her research focuses on global reproductive health and women's empowerment, including human trafficking, HIVIAIDS prevention, and family planning. Other research interests include community participatory research, border health, and mental health. Dr. Withers is the editor of two books: Global Perspectives on Sexual and Reproductive Health Across the Lifecourse, and Global Health Leadership: Case Studies from the Asia-Pacific. She has also published more than 40 scientific articles and serves on the editorial boards of six international global health journals. She spent several years living and working in countries in Asia, Latin America, and subSaharan Africa. 